\section{ASSESSMENT OF THE PROGNOSTIC ROLE OF REGRESSION IN PRIMARY CUTANEOUS MELANOMA}

Karina Aivazian $^{1,2}$, Louise Jackett ${ }^{1,2}$, Serigne Lo $^{2}$, Robert V. Rawson $^{1,2}$, James S. Wilmott ${ }^{2,3}$, Peter M. Ferguson ${ }^{1,2}$, John F. Thompson ${ }^{1,2,3}$, Georgina V. Long ${ }^{2,3}$, Richard A. Scolyer ${ }^{1,2,3}$ ${ }^{1}$ Royal Prince Alfred Hospital, Sydney, Australia; ${ }^{2}$ Melanoma Institute Australia, The University of Sydney, North Sydney, Australia; and ${ }^{3}$ Faculty of Medicine and Health, The University of Sydney, Sydney, Australia

Regression in melanoma is an immunological phenomenon that results in partial or complete disappearance of the tumour. The associations between regression, sentinel lymph node (SLN) status and prognosis, and its implications for treatment, have been questioned for many years. Currently Breslow thickness, tumour mitotic rate, and ulceration are the most important characteristics of primary melanomas for predicting SLN status, which in turn is the most powerful predictor of melanoma survival and is critical for determining appropriate therapy and clinical trial eligibility. It has been suggested recently that regression status may help refine patient selection for SLN biopsy. With this in mind, we are investigating the association of regression in primary melanoma with SLN status and survival by analysing prospectively collected data from the Melanoma Institute Australia database. Results will be presented. As far as we are aware, this will be the largest study of its kind undertaken to date.

\section{FOLLICULAR LYMPHOMA (WHO GRADE 2) OF THE PAROTID GLAND}

Rolla Alaaraj, Simon Nazaretian

Anatomical Pathology Department, Australian Clinical Labs, Clayton, Vic, Australia

Lymphomas arising in salivary glands are rare accounting for less than $5 \%$ of lymphomas overall and constitute $1.7-5 \%$ of all salivary gland neoplasms. The parotid gland is the most commonly involved salivary gland $(70 \%)$. Among lymphomas originating from salivary glands, the ratio of follicular lymphoma is very low. This is a case of a 73-year-old female patient who presented with a parotid mass with a past history of breast cancer. The patient underwent parotidectomy. Macroscopically, there was a well circumscribed white to red nodule up to $18 \mathrm{~mm}$. Microscopically, the salivary gland contained a large lymphoid nodule with well developed architecture, which consisted of variably sized nodules of atypical lymphoid cells. There were 7 centroblasts per 10 high-power field. Immunohistochemistry was performed. The atypical lymphoid cells were positive for CD20, PAX5, BCL2 and CD10 and they were negative for CD5, CD23 and CD3. Features were consistent with follicular lymphoma, WHO grade 2 with Ki-67 proliferative index of 55\%. Follicular lymphoma has an unpredictable, prolonged clinical course with indolent behaviour. There is tendency to transform to a higher grade lymphoma. Although various treatment modalities are used to treat follicular lymphoma, none of them is curative. This necessitates prolonged follow up.

\section{CASE REPORT AND LITERATURE REVIEW OF BURULI ULCER (BU): AN EMERGING DISEASE IN AUSTRALIA CAUSED BY MYCOBACTERIAL ULCERANS SKIN INFECTION}

Rolla Alaaraj $^{1}$, Sureshni I. Jayasinghe ${ }^{1,2}$

${ }^{I}$ Department of Anatomical Pathology, Australian Clinical Labs, Melbourne, Vic, Australia; and ${ }^{2}$ Department of Clinical Pathology, Melbourne Medical School, The University of Melbourne, Melbourne, Vic, Australia

A skin biopsy was received from a 68-year-old female who presented with a non-healing ulcer on the buttock.

Microscopically, ulcerated skin was seen with underlying necrotic tissue and patchy active chronic inflammation. ZiehlNeelsen and Wade-Fite stains demonstrated abundant acid-fast bacilli in and around the ulcer site, consistent with BU.

$\mathrm{BU}$ is an indolent skin infection caused by Mycobacterial ulcerans. People of any age can get infected, and symptoms may occur from 4 weeks to 10 months after exposure. The method of transmission is unclear. Three clinical stages described are: preulcerative, ulcerative and healed disease. PCR or microbiology confirmation is usually necessary for treatment. The first line treatment for early lesions is a combination of antibiotics while surgery is reserved for refractory cases.

The disease exists in 31 countries worldwide, mostly in West and Central Africa. There has been a steady increase in BU notifications since 2015 in Australia. Most cases were encountered in parts of Victoria and Queensland, with a few sporadic cases in Northern Territory, Western Australia and New South Wales. Awareness among pathologists is important to perform special histochemical stains for Mycobacteria in persisting ulcers, nodules, papules, oedema or cellulitis, especially on exposed parts of the body.

\section{MUCINOUS ADENOCARCINOMA OF PROSTATE (MAOP), A RARE VARIANT OF PROSTATE ADENOCARCINOMA: A CASE REPORT AND LITERATURE REVIEW}

Rolla Alaaraj $^{1}$, Sureshni I. Jayasinghe ${ }^{1,2}$

${ }^{I}$ Department of Anatomical Pathology, Australian Clinical Labs, Melbourne, Vic, Australia; and ${ }^{2}$ Department of Clinical Pathology, Melbourne Medical School, The University of Melbourne, Melbourne, Vic, Australia

A prostatectomy specimen was received from a 72-year-old male with a previous needle core biopsy diagnosis of prostatic adenocarcinoma, Gleason score $3+4=7$.

Microscopic examination of the prostatectomy specimen demonstrated single and fused neoplastic glands floating in copious amounts of extracellular mucin representing $>50 \%$ of the tumour volume. There was a minor component of nonmucinous conventional adenocarcinoma in the background. Immunhistochemistry NKX 3.1 and PSA showed positive staining of tumour cells. Clinical, radiological or serological evidence of carcinoma of another site was not revealed. A diagnosis of MAOP, Gleason score 4+3 $=7$ (WHO/ISUP Grade Group 3) with a tertiary component of Gleason pattern 5 comprising $<1 \%$ of total tumour volume was made. 